\title{
Metastasizing Esthesioneuroblastoma in a Dog
}

Veterinary Pathology

2015, Vol. 52(4) 692-695

(C) The Author(s) 2014

Reprints and permission:

sagepub.com/journalsPermissions.nav DOI: 10.1 I77/03009858/4559402

vet.sagepub.com

\author{
K. Siudak', M. Klingler' ${ }^{2}$ M. J. Schmidt' ${ }^{2}$ and C. Herden'
}

$\Theta$

\begin{abstract}
A 7-year-old Afghan hound presented with a history of disorientation, loss of vision, and seizures. Magnetic resonance imaging helped identify a mass at the level of the main olfactory bulb that compressed and displaced adjacent tissues in the cribriform plate into the nasal cavity and nasopharynx. Bony structures were osteolytic. After removing almost $80 \%$ of the mass, the tumor recurred a few months later. Due to severe respiratory distress and subsequent to an ultrasound diagnosis of a liver tumor, the dog was euthanized. In addition to the nasal mass, a single nodule in the liver and multiple nodules in the lung were present. All masses had similar cell morphology and were diagnosed as metastasizing esthesioneuroblastoma. The neoplastic cells expressed neuron-specific enolase and chromogranin $A$, and a few cells within the nasal mass were positive for cytokeratin. This is the first description of a canine esthesioneuroblastoma with distant metastases.
\end{abstract}

\section{Keywords}

esthesioneuroblastoma, metastasis dog, immunohistochemistry, olfactory neuroblastoma

Olfactory neuroblastoma or esthesioneuroblastoma is a rare malignant neoplasm arising in the nasal cavity of humans and domestic animals. ${ }^{8,13}$ Cases in cats, dogs, horses, a heifer, and a monkey have been described. $1,3,4,7,10,12,18$

The neoplastic cells presumably originate from neuroepithelial precursor cells and are typically located in close proximity to the cribriform plate. ${ }^{2}$ Invasive growth leads to osteolysis and deformation of the ethmoturbinates. Metastases to regional lymph nodes, choroid, and retina have been described. ${ }^{7,10,16}$ Metastases to any other peripheral organs are lacking.

This report presents the first description of distant metastases (lung, liver) of a canine esthesioneuroblastoma.

\section{Case History}

A 7-year-old male Afghan hound race dog was presented to the Klinik für Kleintiere, Chirurgie, Justus Liebig Universität (JLU) of Gießen due to disorientation, loss of vision, listlessness, and seizures. Neurologic examination revealed bilateral ventral strabismus, loss of the menace reflex on the right side, pain on palpation of the lumbar region, and a bilateral hypermetric knee-jerk reaction.

Magnetic resonance imaging (MRI) of the head revealed an ovoid, moderately heterogeneous, nasal mass at the level of the right side of the cribriform plate, accompanied by osteolysis. The tumor extended through the nasal cavity into the caudal nasopharynx. The adjacent tissue, especially parts of the main olfactory bulb and the lobus frontalis, was compressed; both cerebral hemispheres and the cerebellum were displaced caudally. Approximately $80 \%$ of the tumor mass was removed surgically.
The remaining neoplastic tissue was treated with irradiation (a total of $40 \mathrm{~Gy}$ ). None of the preexisting clinical signs recurred after treatment. Almost 8 months later, the patient re-presented with severe respiratory distress due to continuous regrowth of the nasal mass. Ultrasound examination revealed a nonhomogeneous mass in the liver. Due to a poor prognosis, the dog was euthanized and necropsied.

\section{Materials and Methods}

Tissue specimens were fixed in $10 \%$ nonbuffered formalin for 2 to 3 days, embedded in paraffin wax, sectioned at approximately $5 \mu \mathrm{m}$, and stained with hematoxylin and eosin.

Immunohistochemistry for vimentin, synaptophysin, S100 protein (S100), glial fibrillary acidic protein (GFAP), neurofilament protein, cytokeratin, neuron-specific enolase (NSE), neuronal nuclear antigen $(\mathrm{NeuN})$, and chromogranin A was performed (Suppl. Table S1). Positive controls for each marker were run simultaneously.

The slides were deparaffinized in xylene substitute (RotiHistol; Carl Roth GmbH \& Co KG, Karlsruhe, Germany) and rehydrated via a descending alcohol series. Endogenous

\footnotetext{
'Institut für Veterinär-Pathologie, Universität Gießen, Germany

${ }^{2}$ Klinik für Kleintiere, Chirurgie, Universität Gießen, Germany

Supplemental material for this article is available on the Veterinary Pathology website at http://vet.sagepub.com/supplemental.

Corresponding Author:

K. Siudak, Institut für Veterinär-Pathologie, Justus-Liebig-Universität Gießen, Frankfurter Str. 96, 35392 Gießen, Germany.

Email: Krystyna.Siudak@vetmed.uni-giessen.de
} 


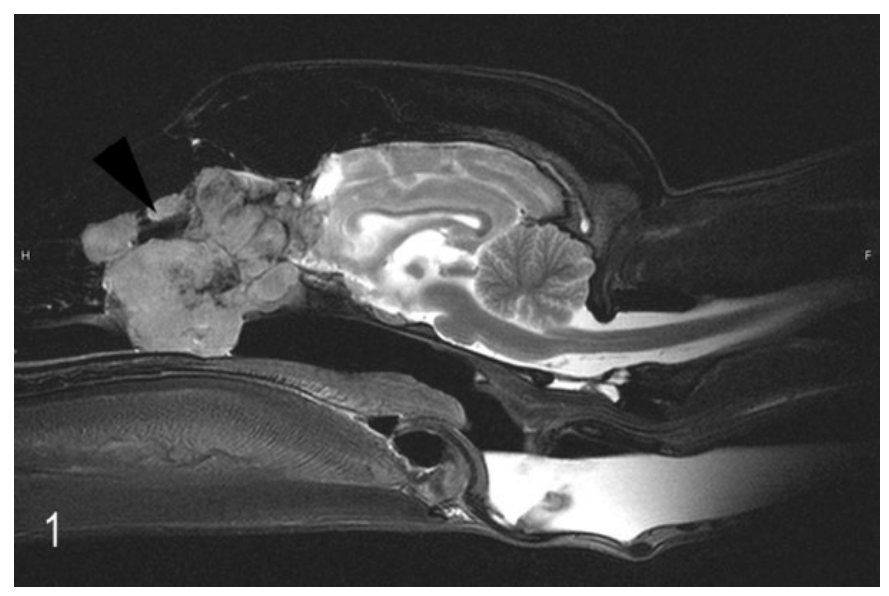

Figure I. Esthesioneuroblastoma, nasal cavity; Afghan hound. Neoplastic mass in the nasal cavity at the level of the cribriform plate (arrow) with invasion into the bulbus olfactorius. Magnetic resonance imaging.

peroxidase activity was inhibited by incubation in methanol with freshly added $0.5 \%$ hydrogen peroxide for 30 minutes at room temperature. Further details regarding the pretreatments are listed in Supplemental Table S1. The sections were incubated overnight with the primary antibodies at $4^{\circ} \mathrm{C}$. For the detection of vimentin, synaptophysin, neurofilament protein, cytokeratin, NSE, and NeuN, a biotinylated secondary horse anti-mouse antibody (Vector Laboratories, Burlingame, CA) was used according to the $\mathrm{ABC}$ staining method $(1: 100,30$ minutes at room temperature). Simultaneously, a secondary swine antirabbit IgG $\mathrm{H}+\mathrm{L}$ antibody (DAKO, Hamburg, Germany), diluted in Tris-buffered saline (TBS) containing 20\% swine serum (1:100), was applied for S100, chromogranin A, and GFAP (30 minutes at room temperature) according to the peroxidase anti-peroxidase (PAP) staining method. Detection of antibody binding was achieved by a peroxidase-based detection system (Vectastain ABC kit standard; Vector Laboratories). Visualization of antibody binding was achieved by adding 3,3'-diaminobenzidine (DAB; Sigma-Aldrich GmbH, Taufkirchen, Germany) as chromogen for 10 minutes. Counterstaining was carried out with Papanicolaou solution (1:20; Merck, Darmstadt, Germany) for 30 seconds. After dehydration, all slides were mounted with a xylene-based solution.

\section{Pathologic Findings}

The mass within the nasal cavity was oval, $5 \times 3 \times 3 \mathrm{~cm}$, and compressed the intracranial region of the main olfactory bulb (Figs. 1, 2). The surface was irregular and covered by a thin layer of brown mucus.

The liver had a single, beige mass $15 \mathrm{~cm}$ in diameter in the right lobe with central dimpling (Fig. 3). Multiple, small nodules were randomly distributed throughout the lung. They measured up to $1 \times 0.5 \times 0.5 \mathrm{~cm}$, and their cut surfaces were white and firm. Both apical lung lobes had few, small, firm, dark red lesions.
The frontal cortex had marked astrogliosis and macrophage infiltration within the meninges at the region of the main olfactory bulb. Samples of the nasal mass harvested at surgery and necropsy, the mass within the liver, and the small nodules within the lung were composed of uniform neoplastic cells with similar morphologic features. The masses were moderately cellular and infiltrative. Nests of tumor cells were unencapsulated but well demarcated by moderate amounts of fibrovascular stroma. The nests were composed of small cuboidal to columnar cells mixed with some spindloid cells (Figs. 4, 5). Occasionally, they had a glandular or palisading pattern. Cells had small to moderate amounts of eosinophilic, homogeneous cytoplasm with indistinct cell borders. Nuclei were round, hyperchromatic, and often basally located in the columnar cells. Nucleoli were inconspicuous. Mitotic figures were rare, less than one per $400 \times$ field. Pseudorosettes (Homer-Wright rosettes) were frequently observed in all neoplastic tissues (Fig. 5). Extensive areas of necrosis were randomly located throughout the neoplasms. A mild infiltration of inflammatory cells, mainly lymphocytes, and a few foci of hemorrhage and numerous siderophages were present in outer regions of the masses, as well as small areas of dystrophic calcification. Tumor cells expressed NSE and chromogranin A in the nasal cavity, liver, and lung (Figs. 6, 7). Neoplastic cells did not express vimentin, synaptophysin, neurofilament, NeuN, S100, or GFAP. Single neoplastic cells of the nasal cavity expressed cytokeratin, whereas no cytokeratin expression was present in any metastatic masses (Suppl. Table S2). Apical lung lobes had mild suppurative bronchopneumonia.

\section{Discussion}

Malignant tumors of the nasal cavity in dogs are rare and are mostly carcinomas followed by different types of sarcomas. ${ }^{8}$ Esthesioneuroblastomas occur rarely and share histologic features with neuroendocrine carcinomas. ${ }^{17}$ Discrimination between tumor types can be problematic. Nasal neuroendocrine carcinomas likely arise from neuroendocrine cells dispersed within the entire sinonasal area; therefore, restriction of tumor to the cribriform plate as present in our case suggests a diagnosis of esthesioneuroblastoma. ${ }^{11,17}$ Invasive tumor growth, in combination with destruction of adjacent bony structures, is most commonly present in esthesioneuroblastomas. ${ }^{13}$ Both neuroendocrine tumors and esthesioneuroblastomas may have rosettes and palisading cells. The expression of neuroendocrine markers such as NSE or chromogranin is indicative but not absolutely diagnostic for esthesioneuroblastomas. ${ }^{17}$ Calcification and necrosis are infrequently present in canine esthesioneuroblastomas. ${ }^{2}$ In combination, these features are of limited diagnostic value. Unfortunately, precise immunohistologic differentiation is hampered by the inconsistent expression pattern of neuroglial markers in both tumor types. ${ }^{17}$ Esthesioneuroblastomas can express NSE, S-100 protein, GFAP, and, less frequently, chromogranin, class III $\beta$-tubulin, microtubule-associated protein 2 (MAP-2), synaptophysin, neurofilament protein, NeuN, and cytokeratin. ${ }^{2,17}$ In comparison, neuroendocrine carcinomas in dogs commonly have 

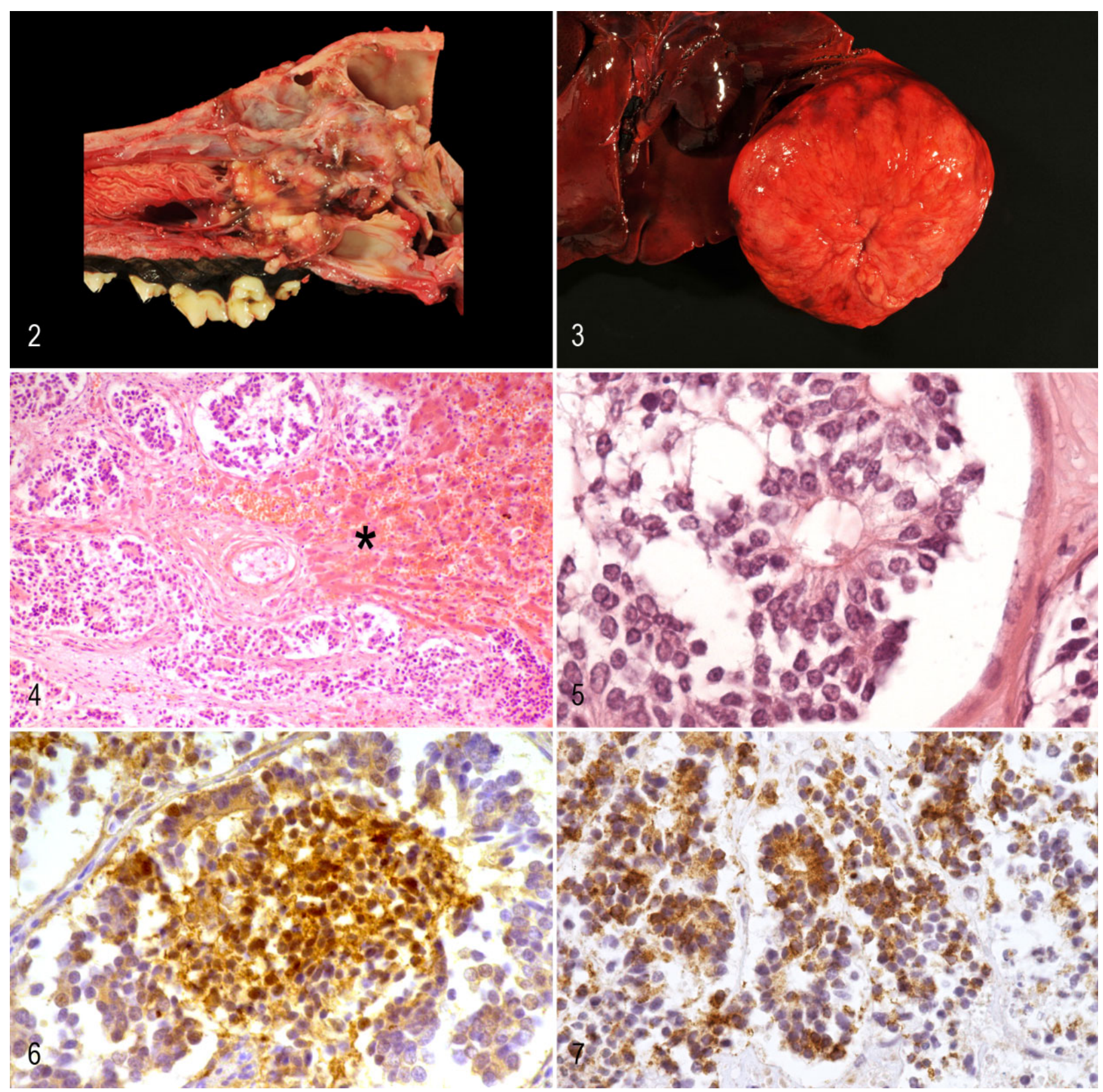

Figure 2. Esthesioneuroblastoma, nasal cavity; dog. An irregular neoplasm obstructs the meatus. Figures 3-4. Esthesioneuroblastoma, liver; dog. Figure 3. A tumor in the right lobe of the liver measures $15 \mathrm{~cm}$ in diameter. Figure 4. Tumor nests, separated by a moderate amount of fibrovascular stroma, surround a vessel. Note the unaffected liver parenchyma (asterisk) with hyperemia. Figures 5-7. Esthesioneuroblastoma, nasal cavity; 7-year-old Afghan hound. Figure 5. Formation of a true rosette (Flexner-Wintersteiner rosette), where cells are arranged around a central lumen. Hematoxylin and eosin. Figure 6. Immunohistochemistry (IHC) shows expression of neuron-specific enolase in tumor cells. Figure 7. IHC for chromogranin A shows positive labeling of tumor cells.

positive immunostaining for cytokeratin AE1/AE3, synaptophysin, NSE, chromogranin A, and S100 as well as vasoactive intestinal peptide (VIP). ${ }^{15}$ The positive reactions for NSE and chromogranin A and the lack of a positive staining for synaptophysin support a diagnosis of esthesioneuroblastoma in our case, although staining for NeuN, neurofilament, S100, and GFAP was not present. Whether this expression pattern, with loss of neuroglial markers, is associated with more aggressive behavior and distant metastases remains unknown. A more intense immunohistochemical staining for cytokeratin is expected in nasal neuroendocrine carcinomas. In the present case, only single cells of the nasal tumor and no cells within the metastases expressed 
cytokeratin. Ultrastructurally, esthesioneuroblastoma should display neural processes containing microtubules. ${ }^{17}$ However, ultrastructural examination was not available for the case presented here. A more detailed histologic grading scheme for esthesioneuroblastomas is available in human medicine. ${ }^{11}$ Four grades are defined around features such as the presence of rosettes, number of mitoses, evidence of necrosis and calcification, and nuclear pleomorphism. Grade III and IV tumors seem to correlate with lower survival times in people. ${ }^{2}$ Applying this grading scheme to our single canine case, the low mitotic index and foci of dystrophic calcification fulfill grade II criteria. The prominent necrosis in many locations of the nasal mass is consistent with grade III and IV tumors. Therefore, this canine esthesioneuroblastoma is classified between grades II and III. It is, however, questionable whether this grading system is relevant to esthesioneuroblastomas in animals. In humans, distant metastases of esthesioneuroblastomas occur occasionally in locations including the cervical lymph nodes, lung, and bone; atypically in the neck; and rarely in the chest wall. ${ }^{5,6,13}$ In people, "drop metastases" are intradural, extramedullary, spinal metastases distal to the primary tumor. They arise subsequent to exfoliation of neoplastic cells into the cerebrospinal fluid of the subarachnoid space. ${ }^{14}$ In animals, esthesioneuroblastomas have been reported in the choroid and retina in a horse and in the regional lymph nodes in dogs. ${ }^{7,10,17}$

In the present case, metastases may have occurred due to a longer survival time than in previously reported cases and perhaps due to a more malignant phenotype. ${ }^{12}$ Also, surgical manipulation could have caused vascular invasion and consequently formation of metastases. Survival is estimated as almost 1 year after radiation therapy. ${ }^{12}$ Median survival time of dogs with tumors in the nasal cavity is approximately 16.5 months. ${ }^{9}$ The lack of distant metastases in animals may be due to euthanasia after initial diagnosis. Furthermore, severe clinical signs and the expense of radiation treatment may shorten survival times. The presence of distant metastases in this case indicates a potentially aggressive behavior of this tumor type in animals. Future study is indicated to more precisely address the biological properties and prognoses of esthesioneuroblastomas.

\section{Acknowledgements}

We thank Diana Klotz and Silke Gantz, Institute of Veterinary Pathology, Justus-Liebig-University of Gießen, for excellent technical support. We also thank Dr Manfred Henrich, Institute of Veterinary Pathology, Justus-Liebig-University of Gießen, for his assistance in adjusting the illustrations.

\section{Author Contribution}

Conception or design: CH. Data acquisition, analysis, or interpretation: KS. Drafting the manuscript: KS. All authors participated in critically revising the manuscript, gave final approval, and agree to be accountable for all aspects of work to ensure integrity and accuracy.

\section{Declaration of Conflicting Interests}

The author(s) declared no potential conflicts of interest with respect to the research, authorship, and/or publication of this article.

\section{Funding}

The author(s) received no financial support for the research, authorship, and/or publication of this article.

\section{References}

1. Anderson BC, Cordy DR. Olfactory neuroblastoma in a heifer. Vet Pathol. 1981;18(4):536-540.

2. Brosinski K, Janik D, Polkinghorne A, et al. Olfactory neuroblastoma in dogs and cats - a histological and immunohistochemical analysis. J Comp Pathol. 2012;146(2-3):152-159.

3. Correa P, Dalgard DW, Adamson RH. Olfactory neuroepithelioma in a cynomolgus monkey (Macaca fascicularis). J Med Primatol. 1975;4(1):51-61.

4. Cox NR, Powers RD. Olfactory neuroblastomas in two cats. Vet Pathol. 1989;26(4):341-343.

5. Dahabreh ID, Janinis D, Stamatelopoulos AG, et al. Surgical resection of esthesioneuroblastoma metastasis to the chest wall. J Thorac Oncol. 2007;2(1):93-95.

6. Davis RE, Weissler MC. Esthesioneuroblastoma and neck metastasis. Head Neck. 1992;14(6):477-482.

7. Döpke C, Gröne A, von Borstel M, et al. Metastatic esthesioneuroblastoma in a horse. J Comp Pathol. 2005;132(2-3):218-222.

8. Dungworth DL, Hauser B, Hahn FF, et al. WHO Histological Classification of Tumors of the Respiratory System of Domestic Animals. 2nd ed., vol VI. Washington, DC: Armed Forces Institute of Pathology; 1999:16-21.

9. Evans SM, Goldschmidt M, McKee LJ, et al. Prognostic factors and survival after radiotherapy for intranasal neoplasms in dogs: 70 cases (1974-1985). J Am Vet Med Assoc. 1989;194(10): 1460-1463.

10. Hara K, Shimada A, Morita T, et al. Olfactory neuroepithelioma in a dog: an immunohistochemical and electron microscopic study. J Vet Med Sci. 2002;64(4):391-393.

11. Hyams VJ, Batsakis JG, Michaels L. Tumors of the upper respiratory tract and ear. In: Hyams VJ, ed. Atlas of Tumor Pathology. 2nd series, fascicle 25. Washington, DC: Armed Forces Institute of Pathology; 1988:240-248.

12. Kitagawa M, Okada M, Yamamura H, et al. Diagnosis of olfactory neuroblastoma in a dog by magnetic resonance imaging. Vet Rec. 2006;159(9):288-289.

13. Pickuth D, Heywang-Köbrunner SH. Imaging of recurrent esthesioneuroblastoma. Br J Radiol. 1999;72(863):1052-1057.

14. Rao AJ, Gultekin SH, Neuwelt EA, et al. Late occurrence of drop metastasis to the spine in a case of esthesioneuroblastoma. J Neurosurg Spine. 2011;15(5):571-575.

15. Sako T, Shimoyama Y, Akihara Y, et al. Neuroendocrine carcinoma in the nasal cavity of ten dogs. J Comp Pathol. 2005;133:155-163.

16. Schrenzel MD, Higgin RJ, Hinrichs $\mathrm{SH}$, et al. Type $\mathrm{C}$ retroviral expression in spontaneous feline olfactory neuroblastomas. Acta Neuropathol. 1990;80:547-553.

17. Wilson DW, Dungworth DL. Tumors in domestic animals. In: Meuten DJ, ed. Tumors in Domestic Animals. 4th ed. Ames: Iowa State University Press; 2002:365-400.

18. Yamate J, Izawa T, Ogata K, et al. Olfactory neuroblastoma in a horse. J Vet Med Sci. 2006;68(5):495-498. 